\begin{tabular}{|c|c|}
\hline Title & Josephson effect in noncentrosy mmetric superconductor junctions \\
\hline Author(s) & A sano, $Y$ asuhiro; $Y$ amano, Satoshi \\
\hline Citation & $\begin{array}{l}\text { Physical Review B, 84(6), } 064526 \\
\text { https://doi.org/10.1103/PhysRevB.84.064526 }\end{array}$ \\
\hline Issue Date & $2011-08-01$ \\
\hline Doc URL & http:/hdl .handle.net/2115/47110 \\
\hline Rights & @2011 A merican Physical Society \\
\hline Type & article \\
\hline File Information & PRB84-6_064526.pdf \\
\hline
\end{tabular}

Instructions for use 


\title{
Josephson effect in noncentrosymmetric superconductor junctions
}

\author{
Yasuhiro Asano ${ }^{1}$ and Satoshi Yamano ${ }^{2}$ \\ ${ }^{1}$ Department of Applied Physics and Center for Topological Science \& Technology, Hokkaido University, Sapporo 060-8628, Japan \\ ${ }^{2}$ Department of Applied Physics, Hokkaido University, Sapporo 060-8628, Japan
}

(Received 28 April 2011; revised manuscript received 13 July 2011; published 25 August 2011)

\begin{abstract}
We discuss the Josephson current between two noncentrosymmetric superconductors. The coexistence of superconducting order parameters between the spin-singlet $\Delta_{\mathrm{S}}$ and the helical $p$-wave spin-triplet $\Delta_{\mathrm{T}}$ enriches a variety of low-temperature behavior of the Josephson current depending on their relative amplitudes. We will show that characteristic behaviors of the Josephson current for $\Delta_{\mathrm{S}}>\Delta_{\mathrm{T}}$ are clearly different from those for $\Delta_{\mathrm{S}}<\Delta_{\mathrm{T}}$. The topologically protected zero-energysurface bound states are responsible for the clear difference. We conclude that the Josephson current reflects well the character of the topological surface states and the pairing symmetry of noncentrosymmetric superconductors.
\end{abstract}

DOI: 10.1103/PhysRevB.84.064526 PACS number(s): 74.25.nn, 74.45.+c, 74.78.Fk, 74.70.-b

\section{INTRODUCTION}

The coexistence of the spin-singlet superconducting order parameters and the spin-triplet one is the essential feature of noncentrosymmetric superconductors (NCSs). ${ }^{1-4}$ The absence of spatial inversion symmetry leads to spin-orbit coupling large enough to mix the spin-singlet component and the spin-triplet component. The amplitude of the spin-singlet component $\Delta_{\mathrm{S}}$ and that of the spin-triplet component $\Delta_{\mathrm{T}}$ is a material parameter determined by the amplitude of spin-orbit coupling. The Rashba-type spin-orbit coupling induces the helical $p$-wave spin-triplet order parameter, which is the topologically nontrivial superconducting state. ${ }^{4,5}$ There have been several studies on coexistent superconducting properties of the spin-singlet $s$-wave and the spin-triplet helical $p$-wave symmetries. ${ }^{6-12} \mathrm{It}$ is known that topologically protected states with linear dispersion appear at a surface of a NCS for $\Delta_{T}>\Delta_{S}$. Recent papers, however, have suggested mixed order-parameter spin-singlet $d$-wave and spin-triplet $p$-wave symmetries ${ }^{13,14}$ that have been proposed for the interfacial superconductivity. ${ }^{15}$ Such pairing symmetry results in a dispersionless surface bound state at the Fermi level. A similar flat zero-energy-surface state in a NCS has also been discussed very recently. ${ }^{16}$ Physical values originated from the bulk region of a superconductor such as the specific heat and spin susceptibility ${ }^{4}$ are expected to be interpolated from those in the two limits: the pure spin-singlet case and the pure spin-triplet one. An open question is how physical values governed by the surface bound states behave as a function of the relative amplitude between $\Delta_{T}$ and $\Delta_{S}$. This paper addresses this issue. A very resent paper has commented on the drastic change of the tunneling spectra of NCS depending of the relative amplitudes. ${ }^{17}$

The surface bound states of unconventional superconductors have been theoretically discussed in heavy fermionic superconductors, ${ }^{18}$ the polar state of ${ }^{3} \mathrm{He},{ }^{19}$ and high- $T_{c}$ cuprates. $^{20,21}$ Experimentally, the presence of the surface bound states has been observed as the zero-bias anomaly ${ }^{21,22}$ of the scanning tunneling spectroscopy (STS) of hole-doped ${ }^{23,24}$ and electron-doped ${ }^{25}$ high- $T_{c}$ cuprates. The zero-bias anomaly has been observed also in the differential conductance of ramp-edge junctions of hole-doped high- $T_{c}$ cuprates $^{26}$ and the grain-boundary junction of electron-doped high- $T_{c}$ cuprates. ${ }^{27}$ The presence of the surface bound states has been reinterpreted since the proposal for new classification of matter. ${ }^{28}$ The surface bound states at zero energy are necessary to naturally connect a nontrivial topological integer number inside of an unconventional superconductor with the trivial topological number outside of the superconductor. The dispersion of the subgap states depends on the type of the topological number defined in superconductors. Chiral or helical superconductors give rise to dispersive surface bound states. ${ }^{29}$ On the other hand, dispersionless zero-energy states are formed under $d_{x^{2}-y^{2}}$ and $p_{x}$-wave symmetries.

In the direct current Josephson effect, the surface bound states result in large $J_{c} R_{N} /\left(\Delta_{0} / e\right)$ values and the deviation of the current-phase relationship from the sinusoidal function at low temperature, ${ }^{30,31}$ where $J_{c}$ is the critical Josephson current, $R_{N}$ is the normal resistance of a junction, and $\Delta_{0}$ is the amplitude of pair potential at zero temperature. Such behavior is called the low-temperature anomaly of the Josephson current and is known to be sensitive to spectra of surface bound states. ${ }^{30-34}$ So far the Josephson effect between an $s$-wave superconductor and a $\mathrm{NCS}^{35}$ and that between two $\mathrm{NCSs}^{36}$ have been studied based on the tunneling Hamiltonian method. The low-temperature anomaly of the Josephson current has not yet been discussed.

In this paper, we theoretically calculate the Josephson current between two NCSs ${ }^{37,38}$ based on a current formula ${ }^{39}$ in terms of the Andreev reflection coefficients of junctions. We assume an order parameter that is a mixture of the spin-singlet $s$-wave and the spin-triplet helical $p$-wave symmetries. We call such a state an $s+p$ mixture. For $\Delta_{\mathrm{T}}<\Delta_{\mathrm{S}}$, the Josephson current saturates at low temperature as described by the Ambegaokar-Baratoff formula. ${ }^{40}$ On the other hand, for $\Delta_{\mathrm{T}}>$ $\Delta_{S}$, the Josephson current increases logarithmically with decreasing temperature $(T)$. The interfacial bound state causes the low-temperature anomaly. The characteristic behavior of the Josephson current does not change gradually as a function of the relative amplitude between $\Delta_{\mathrm{T}}$ and $\Delta_{\mathrm{S}}$. The critical point $\Delta_{\mathrm{T}}=\Delta_{\mathrm{S}}$ clearly divides the qualitative feature of the Josephson current.

In addition to the $s+p$ mixture, we also consider two types of mixed order parameters between the spin-singlet $d_{x y}$-wave and the spin-triplet helical $p$-wave symmetries. We call such a state a $d+p$ mixture. The features of the Josephson current are 


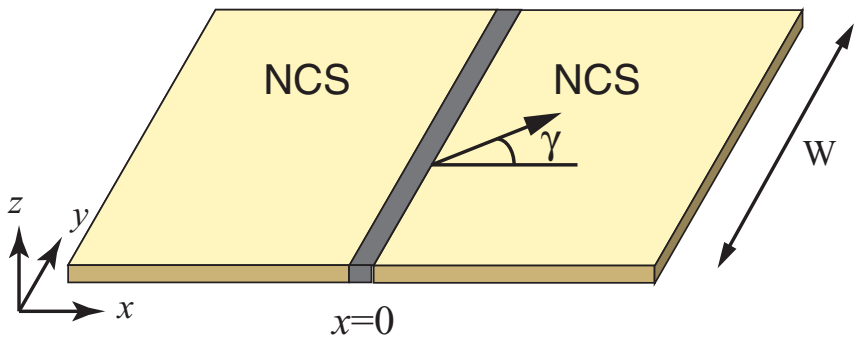

FIG. 1. (Color online) Schematic picture of the Josephson junction.

well characterized by the relative amplitude between $\Delta_{\mathrm{T}}$ and $\Delta_{\mathrm{S}}$. In some cases, the Josephson current increases as $1 / T$ with decreasing temperature due to the dispersionless zero-energy state. We will discuss the physics behind such clear qualitative changes of the Josephson effect in terms of topologically protected zero-energy surface states. It is known that the excitation of such surface bound states on superconductors is characterized by the Majorana fermion. ${ }^{29,41,42}$ Unusual phenomena peculiar to the Majorana fermion have been suggested theoretically. ${ }^{43-45}$

This paper is organized as follows. In Sec. II, we discuss a theoretical model of the Josephson junction consisting of two NCSs. In Sec. III, we show the calculated results of the Josephson current for $s+p$ and $d+p$ mixtures. We summarize this paper in Sec. IV.

\section{MODEL}

Let us consider a Josephson junction between two NCSs as shown in Fig. 1, where the electric current flows in the $x$ direction and the junction width in the $y$ direction is $W$. We apply a periodic boundary condition in the $y$ direction. The Bogoliubov-de Gennes (BdG) Hamiltonian in momentum space reads

$$
H_{\mathrm{BdG}}(\boldsymbol{k})=\left[\begin{array}{cl}
\hat{h}(\boldsymbol{k}) & \hat{\Delta}(\boldsymbol{k}) \\
-\hat{\Delta}^{*}(-\boldsymbol{k}) & -\hat{h}^{*}(-\boldsymbol{k})
\end{array}\right],
$$

$$
\begin{gathered}
\hat{h}(\boldsymbol{k})=\xi_{\boldsymbol{k}} \hat{\sigma}_{0}+\lambda \boldsymbol{g} \cdot \hat{\boldsymbol{\sigma}}, \\
\xi_{\boldsymbol{k}}=\frac{\hbar^{2} \boldsymbol{k}^{2}}{2 m}-\mu,
\end{gathered}
$$

where $\hat{\sigma}_{j}$ for $j=1-3$ are the Pauli matrices, $\hat{\sigma}_{0}$ is the unit matrix in spin space, $k_{x(y)}$ is the wave number in the $x(y)$ direction, $k_{F}$ is the Fermi wave number, $\mu$ is the chemical potential, and $\lambda$ is the amplitude of the spin-orbit interaction. In this paper, we assume that $\lambda \ll \mu$. We consider the Rashba-type spin-orbit coupling reflecting the noncentrosymmetry along the $z$ direction [i.e., $\boldsymbol{g}=\left(k_{y},-k_{x}, 0\right) / k_{F}$ ]. Correspondingly, we choose the $\boldsymbol{d}$ vector in the pair potential as $\boldsymbol{d}=\boldsymbol{g}$ as discussed in Ref. 4. As a consequence, the spin-triplet part of the pair potential has helical $p$-wave symmetry. In this paper, we consider three types of mixed order parameters, as follows:

$$
\hat{\Delta}(\boldsymbol{k})=\left\{\begin{array}{cc}
i\left(\Delta_{\mathrm{T}} \boldsymbol{d} \cdot \boldsymbol{\sigma}+\Delta_{\mathrm{S}}\right) \hat{\sigma}_{2}, & s+p, \\
i\left(\Delta_{\mathrm{T}} \boldsymbol{d} \cdot \boldsymbol{\sigma}+\Delta_{\mathrm{S}} \sin 2 \gamma\right) \hat{\sigma}_{2}, & d+p \mathrm{I}, \\
i\left(\Delta_{\mathrm{T}} \boldsymbol{d} \cdot \boldsymbol{\sigma}+\Delta_{\mathrm{S}}\right) \sin 2 \gamma \hat{\sigma}_{2}, & d+p \mathrm{II},
\end{array}\right.
$$

where $\gamma$ is the incident angle of a quasiparticle as shown in Fig. 1 and $e^{i \gamma}=\left(k_{x}+i k_{y}\right) / k_{F}$. The first one consists of $s$-wave singlet and helical $p$-wave triplet components. The pair potential of $d+p$ II is the order parameter discussed in interfacial superconductivity. ${ }^{14}$ Although the pair potential of $d+p$ I may not have a relation to any real materials, we consider it for theoretical interest. The energy eigenvalues of Eq. (1) are $E= \pm E_{ \pm}$with $E_{ \pm}=\sqrt{\left(\xi_{k} \pm \lambda\right)^{2}+\Delta_{ \pm}^{2}}$ and

$$
\Delta_{ \pm}(\gamma)=\left\{\begin{array}{cc}
\Delta_{\mathrm{S}} \pm \Delta_{\mathrm{T}}, & s+p, \\
\Delta_{\mathrm{S}} \sin 2 \gamma \pm \Delta_{\mathrm{T}}, & d+p \text { I } \\
\left(\Delta_{\mathrm{S}} \pm \Delta_{\mathrm{T}}\right) \sin 2 \gamma, & d+p \text { II. }
\end{array}\right.
$$

To represent the wave function of a quasiparticle, we need other values of the pair potential defined by $\tilde{\Delta}_{ \pm}(\gamma)=\Delta_{ \pm}(\pi-\gamma)$,

$$
\tilde{\Delta}_{ \pm}(\gamma)=\left\{\begin{array}{cc}
\Delta_{\mathrm{S}} \pm \Delta_{\mathrm{T}}, & s+p, \\
-\Delta_{\mathrm{S}} \sin 2 \gamma \pm \Delta_{\mathrm{T}}, & d+p \text { I, } \\
-\left(\Delta_{\mathrm{S}} \pm \Delta_{\mathrm{T}}\right) \sin 2 \gamma, & d+p \text { II. }
\end{array}\right.
$$

The wave function in the left superconductor is obtained as

$$
\begin{aligned}
& \Psi_{L}(x, y)=\check{\Phi}_{L}\left\{\left[\begin{array}{cc}
u_{+} & u_{-} \\
-i e^{i \gamma} u_{+} & i e^{i \gamma_{u_{-}}} \\
i e^{i \gamma} v_{+} & -i e^{i \gamma} v_{-} \\
v_{+} & v_{-}
\end{array}\right]\left[\begin{array}{c}
a_{+} \\
a_{-}
\end{array}\right] e^{i k_{x} x}+\left[\begin{array}{cc}
\tilde{v}_{+} & \tilde{v}_{-} \\
i e^{-i \gamma} \tilde{v}_{+} & -i e^{-i \gamma} \tilde{v}_{-} \\
-i e^{-i \gamma} \tilde{u}_{+} & i e^{-i \gamma} \tilde{u}_{-} \\
\tilde{u}_{+} & \tilde{u}_{-}
\end{array}\right]\left[\begin{array}{l}
b_{+} \\
b_{-}
\end{array}\right] e^{-i k_{x} x}\right. \\
& \left.+\left[\begin{array}{cc}
\tilde{u}_{+} & \tilde{u}_{-} \\
i e^{-i \gamma} \tilde{u}_{+} & -i e^{-i \gamma} \tilde{u}_{-} \\
-i e^{-i \gamma} \tilde{v}_{+} & i e^{-i \gamma} \tilde{v}_{-} \\
\tilde{v}_{+} & \tilde{v}_{-}
\end{array}\right]\left[\begin{array}{c}
A_{+} \\
A_{-}
\end{array}\right] e^{-i k_{x} x}+\left[\begin{array}{cc}
v_{+} & v_{-} \\
-i e^{i \gamma} v_{+} & i e^{i \gamma} v_{-} \\
i e^{i \gamma} u_{+} & -i e^{i \gamma} u_{-} \\
u_{+} & u_{-}
\end{array}\right]\left[\begin{array}{c}
B_{+} \\
B_{-}
\end{array}\right] e^{i k_{x} x}\right\} e^{i k_{y} y} \\
& u_{ \pm}=\sqrt{\frac{1}{2}\left(1+\frac{\Omega_{ \pm}}{E}\right)}, \quad v_{ \pm}=\sqrt{\frac{1}{2}\left(1-\frac{\Omega_{ \pm}}{E}\right)} s_{ \pm}, \quad \tilde{u}_{ \pm}=\sqrt{\frac{1}{2}\left(1+\frac{\tilde{\Omega}_{ \pm}}{E}\right)}, \quad \tilde{v}_{ \pm}=\sqrt{\frac{1}{2}\left(1-\frac{\tilde{\Omega}_{ \pm}}{E}\right)} \tilde{s}_{ \pm} \\
& \Omega_{ \pm}=\sqrt{E^{2}-\Delta_{ \pm}^{2}}, \quad \tilde{\Omega}_{ \pm}=\sqrt{E^{2}-\tilde{\Delta}_{ \pm}^{2}}, \quad s_{ \pm}=\frac{\Delta_{ \pm}}{\left|\Delta_{ \pm}\right|}, \quad \tilde{s}_{ \pm}=\frac{\tilde{\Delta}_{ \pm}}{\left|\tilde{\Delta}_{ \pm}\right|}, \quad \check{\Phi}_{j}=\operatorname{diag}\left\{e^{i \varphi_{j} / 2}, e^{i \varphi_{j} / 2}, e^{-i \varphi_{j} / 2}, e^{-i \varphi_{j} / 2}\right\}
\end{aligned}
$$


where $a_{ \pm}$and $b_{ \pm}$are the amplitudes of incoming waves, $A_{ \pm}$and $B_{ \pm}$are the amplitudes of outgoing waves, and $\varphi_{j}$ for $j=L$ or $R$ is the macroscopic phase of the superconductor. In the same way, the wave function in the right superconductor is represented by

$$
\Psi_{R}(x, y)=\check{\Phi}_{R}\left\{\left[\begin{array}{cc}
u_{+} & u_{-} \\
-i e^{i \gamma} u_{+} & i e^{i \gamma} u_{-} \\
i e^{i \gamma} v_{+} & -i e^{i \gamma} v_{-} \\
v_{+} & v_{-}
\end{array}\right]\left[\begin{array}{c}
C_{+} \\
C_{-}
\end{array}\right] e^{i k_{x} x}+\left[\begin{array}{cc}
\tilde{v}_{+} & \tilde{v}_{-} \\
i e^{-i \gamma} \tilde{v}_{+} & -i e^{-i \gamma} \tilde{v}_{-} \\
-i e^{-i \gamma} \tilde{u}_{+} & i e^{-i \gamma} \tilde{u}_{-} \\
\tilde{u}_{+} & \tilde{u}_{-}
\end{array}\right]\left[\begin{array}{c}
D_{+} \\
D_{-}
\end{array}\right] e^{-i k_{x} x}\right\} e^{i k_{y} y},
$$

with $C_{ \pm}$and $D_{ \pm}$being amplitudes of outgoing waves. At the junction interface, we introduce the potential barrier described by $V_{0} \delta(x)$. Throughout this paper, we fix $z_{0} \equiv$ $\left(V_{0} m\right) /\left(\hbar^{2} k_{F}\right)=5$, which leads to the transmission probability of the insulating barrier $T_{B}=\int_{0}^{\pi / 2} d \gamma \cos ^{3} \gamma /\left(z_{0}^{2}+\cos ^{2} \gamma\right)$ at about 0.01 . By eliminating $C_{ \pm}$and $D_{ \pm}$using a boundary condition, it is possible to obtain the reflection coefficients

$$
\left[\begin{array}{l}
A_{+} \\
A_{-} \\
B_{+} \\
B_{-}
\end{array}\right]=\left[\begin{array}{ll}
\hat{r}_{e e} & \hat{r}_{e h} \\
\hat{r}_{h e} & \hat{r}_{h h}
\end{array}\right]\left[\begin{array}{l}
a_{+} \\
a_{-} \\
b_{+} \\
b_{-}
\end{array}\right]
$$

The Josephson current can be calculated based on a formula ${ }^{39}$ after applying the continuation $E \rightarrow i \omega_{n}=i(2 n+1) \pi T$ :

$$
J=\frac{e}{2 \hbar} \sum_{k_{y}} T \sum_{\omega_{n}} \operatorname{Tr}\left\{\left[\begin{array}{cc}
\frac{\Delta_{+}}{\Omega_{n+}} & 0 \\
0 & \frac{\Delta_{-}}{\Omega_{n-}}
\end{array}\right] \hat{r}_{h e}-\left[\begin{array}{cc}
\frac{\tilde{\Delta}_{+}}{\bar{\Omega}_{n+}} & 0 \\
0 & \frac{\tilde{\Delta}_{-}}{\tilde{\Omega}_{n-}}
\end{array}\right] \hat{r}_{e h}\right\},
$$

with $\Omega_{n \pm}=\sqrt{\omega_{n}^{2}+\Delta_{ \pm}^{2}}$ and $\tilde{\Omega}_{n \pm}=\sqrt{\omega_{n}^{2}+\tilde{\Delta}_{ \pm}^{2}}$. We introduce a parameter $0 \leqslant \alpha \leqslant 1$ to tune the mixing rate between the spin-singlet and spin-triplet components as

$$
\Delta_{\mathrm{S}}=\alpha \Delta, \quad \Delta_{\mathrm{T}}=(1-\alpha) \Delta,
$$

where the dependence of $\Delta$ on temperature $T$ is described by BCS theory. The energy spectra of a subgap state at a surface of the NCS is calculated from Eq. (10) with the boundary condition $\Psi_{R}(0, y)=0$.

\section{RESULTS}

At first, we summarize the energy spectra of the subgap state at the surface of the superconductor in Fig. 2. In the $s+p$ mixture, the energy of the bound state satisfies

$$
\left(E^{2}-\Delta_{+} \Delta_{-}\right) \cos ^{2} \gamma+\Omega_{+} \Omega_{-}\left(1+\sin ^{2} \gamma\right)=0 .
$$

It is known that the surface bound state is absent for $\Delta_{\mathrm{S}}>\Delta_{\mathrm{T}}$, whereas the surface bound states with the linear dispersion exist for $\Delta_{\mathrm{S}}>\Delta_{\mathrm{T}}$, as shown in Fig. 2(a).

In the $d+p$ I mixture, the energy of the surface bound state satisfies

$$
E^{2}\left(1+\cos ^{2} \gamma\right)+\sin ^{2} \gamma\left(\Delta_{+} \Delta_{-}+\Omega_{+} \Omega_{-}\right)=0 .
$$

The equation has two solutions. The first one is $E=0$, which is allowed for $|\sin \gamma| \geqslant \Delta_{\mathrm{T}} / \Delta_{\mathrm{S}}$, as shown in Fig. 2(b). The dispersionless zero-energy bound states are a direct consequence of the $d_{x y}$-wave symmetry. ${ }^{20}$ Therefore such a flat zero-energy state is absent for $\Delta_{\mathrm{S}}<\Delta_{\mathrm{T}}$. The second solution is given by

$$
E= \pm \sin \gamma \sqrt{\Delta_{\mathrm{T}}^{2}-\Delta_{\mathrm{S}}^{2} 4 \sin ^{2} \gamma}
$$

for $|\tan \gamma|<\Delta_{\mathrm{T}} /\left(2 \Delta_{\mathrm{S}}\right)$, as shown in Figs. 2(b) and 2(c).

In the $d+p$ II mixture, the energy of the surface bound states satisfies

$$
E^{2}\left(1+\sin ^{2} \gamma\right)+\cos ^{2} \gamma\left(\Delta_{+} \Delta_{-}+\Omega_{+} \Omega_{-}\right)=0 .
$$

The equation has two solutions. The first one is $E=0$ for all $\gamma$, which is possible only when $\Delta_{\mathrm{S}}>\Delta_{\mathrm{T}}$, as shown in Fig. 2(d). The second solution is given by

$$
E= \pm 2 \cos ^{2} \gamma \sqrt{\Delta_{\mathrm{T}}^{2} \sin ^{2} \gamma-\Delta_{\mathrm{S}}^{2}}
$$

which is allowed for $|\sin \gamma|<\sqrt{\Delta_{\mathrm{S}} / \Delta_{\mathrm{T}}}$, as shown in Fig. 2(e).

$\Delta_{\mathrm{S}}>\Delta_{\mathrm{T}}$ $\Delta_{\mathrm{S}}<\Delta_{\mathrm{T}}$
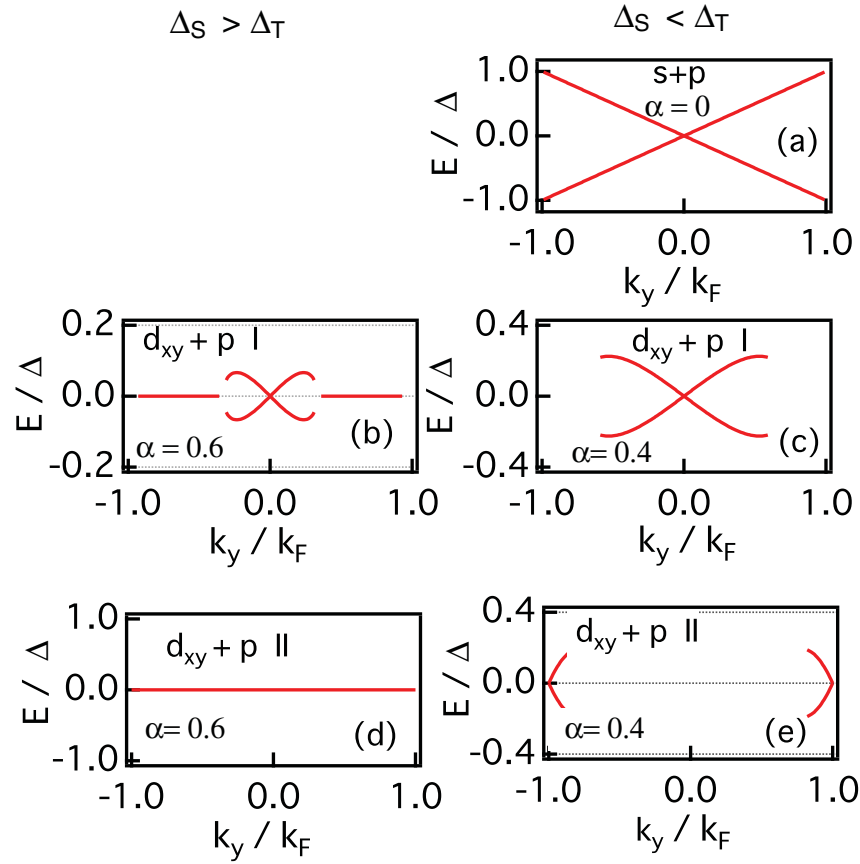

FIG. 2. (Color online) The energy spectra of the surface bound state. (a) $s+p$ at $\alpha=0$, (b) $d+p$ I at $\alpha=0.6$, (c) $d+p$ I at $\alpha=0.4$, (d) $d+p$ II at $\alpha=0.6$, and (e) $d+p$ II at $\alpha=0.4$. The horizontal axis $k_{y} / k_{F}$ corresponds to $\sin \gamma$. 

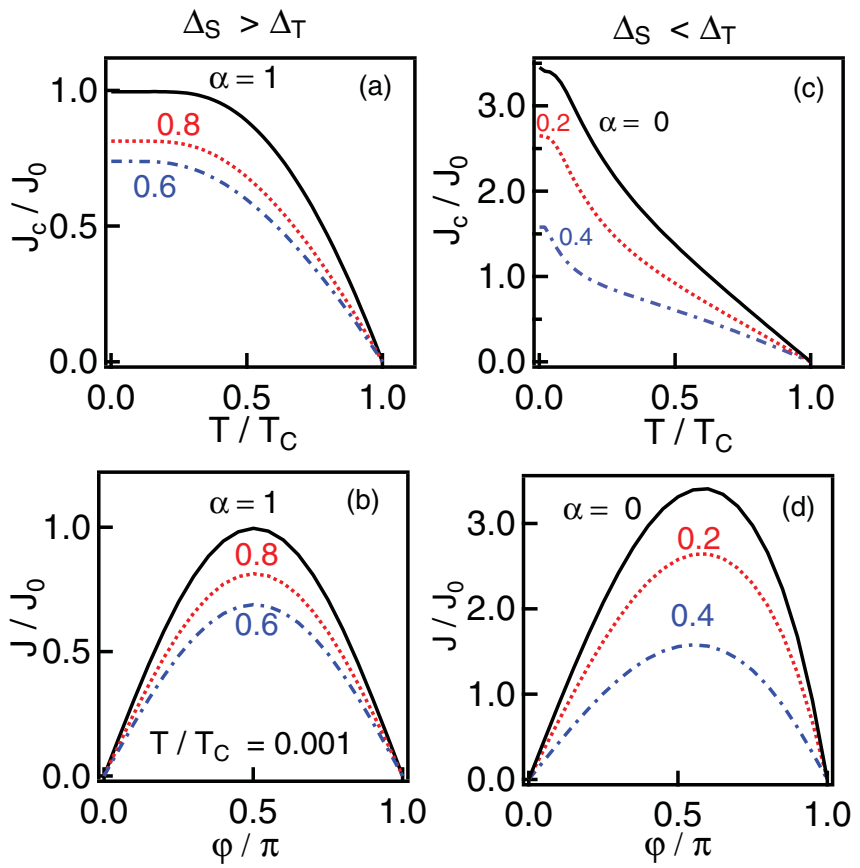

FIG. 3. (Color online) Results for the $s+p$ mixture. The critical Josephson current is plotted as a function of temperature in (a) and (c). The current-phase relationships are shown in (b) and (d) at a low temperature $\left(T=0.001 T_{c}\right.$ ). The transmission probability of the tunnel junction $T_{B}$ is fixed at about 0.01 throughout this paper.

\section{A. $s+p$}

In Fig. 3, we show the calculated results of the Josephson current for the $s+p$ mixture. In Fig. 3(a), we plot the critical Josephson current as a function of temperature for several choices of $\alpha$ satisfying $\Delta_{\mathrm{S}}>\Delta_{\mathrm{T}}$. The Josephson current is normalized by $J_{0}=\pi \Delta_{0} /\left(2 e R_{N}\right)$, where $R_{N}$ is the normal resistance of the junction. In the case of metallic superconductor junctions, the Josephson critical current becomes $J_{0}$ at zero temperature. In Fig 3(b), we show the current-phase relationship (CPR) at a low temperature $T=$ $0.001 T_{c}$ for $\alpha=1,0.8$, and 0.6 , where $\varphi=\varphi_{L}-\varphi_{R}$ is the phase difference across the junction. The critical Josephson current saturates at low temperature and the CPR is sinusoidal for $\alpha=1,0.8$, and 0.6. Thus the Josephson current for $\Delta_{\mathrm{S}}>\Delta_{\mathrm{T}}$ obeys the Ambegaokar-Baratoff relation because there is no surface zero-energy state. In Figs. 3(c) and 3(d), respectively, we show the dependence of critical current on temperature and the CPR at a low temperature for several choices of $\alpha$ satisfying $\Delta_{\mathrm{S}}<\Delta_{\mathrm{T}}$. The results in Figs. 3(c) and 3(d) show qualitatively different behavior from those in Figs. 3(a) and 3(b), respectively. The critical Josephson current in Fig. 3(c) increases with decreasing temperature even far below $T_{c}$. This behavior is called the low-temperature anomaly of the Josephson current. The resonant tunneling through the surface bound state at zero energy is responsible for the anomaly. ${ }^{30,31}$ Such a zero-energy state $(\sin \gamma=0)$ is possible, as shown in Fig. 2(a). According to previous papers, ${ }^{32,33}$ the Josephson critical current increases logarithmically with decreasing temperature for $\alpha=0$. The results for $\alpha=0.2$ and 0.4 also show the logarithmic low-temperature anomaly. Correspondingly, the contribution of higher harmonics slightly
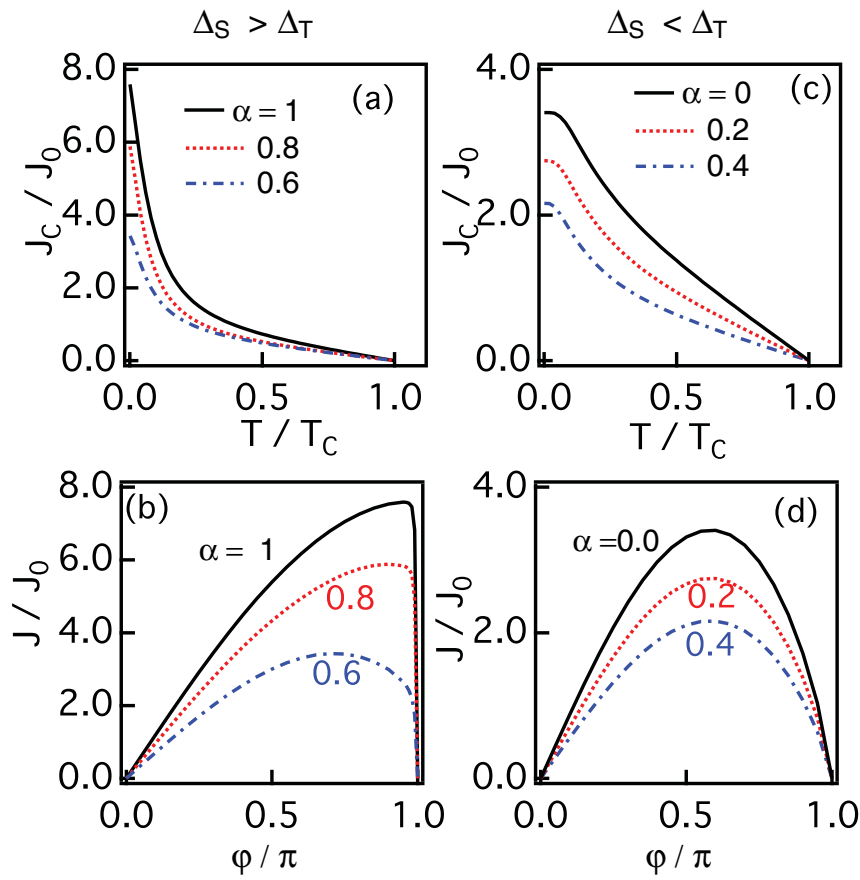

FIG. 4. (Color online) Result for $d+p$ I. The critical Josephson current is plotted as a function of temperature in (a) and (c). The current-phase relationship is shown in (b) and (d) at low temperature $\left(T=0.001 T_{c}\right)$.

deviates the CPR from the sinusoidal relation, as shown in Fig. 3(d). Thus the characteristic feature of Josephson current qualitatively changes at the singular point of $\Delta_{\mathrm{S}}=\Delta_{\mathrm{T}}$.

\section{B. $d+p I$}

Next, we show the calculated results of the Josephson current for the $d+p$ I mixture as shown in Fig. 4. The temperature dependence of critical current for $\alpha=1,0.8$, and 0.6 satisfying $\Delta_{\mathrm{S}}>\Delta_{\mathrm{T}}$ shows a low-temperature anomaly, as shown in Fig. 4(a). The critical current increases as $T^{-1}$ with decreasing temperature at $\alpha=1 .^{30}$ The results for $\alpha=0.8$ and 0.6 also show such a power-law-like low-temperature anomaly. The corresponding CPR shown in Fig. 4(b) indicates a jump at $\varphi=\pi$ because of the contributions of higher harmonics. In this case, the surface bound states are energetically localized at $E=0$, as shown in Fig. 2(b). The resonant tunneling through such zero-energy states causes the strong low-temperature anomaly. For $\Delta_{\mathrm{S}}<\Delta_{\mathrm{T}}$, on the other hand, the Josephson current in Figs. 4(c) and 4(d) has properties similar to those in Figs. 3(c) and 3(d), respectively. The critical current indicates the logarithmic low-temperature anomaly. The presence of the zero-energy surface bound state at $\sin \gamma=0$ in Fig. 2(c) explains the similarity. Thus the characteristic feature of the Josephson current for the $d+p$ I mixture also changes qualitatively around the point of $\Delta_{\mathrm{S}}=\Delta_{\mathrm{T}}$.

The large deviation of the CPR from the sinusoidal function in Fig. 4(b) can be seen only at low temperature. According to an analytical expression of the Josephson current for $\alpha=1$, the higher harmonics contribute to the Josephson current when the temperature is much smaller than $\sqrt{T_{B}} \Delta_{0}$. Here $T_{B}$ is the transmission probability of the tunnel junction and is about 0.01 in the present calculation. In Fig. 5, we show the CPR for 

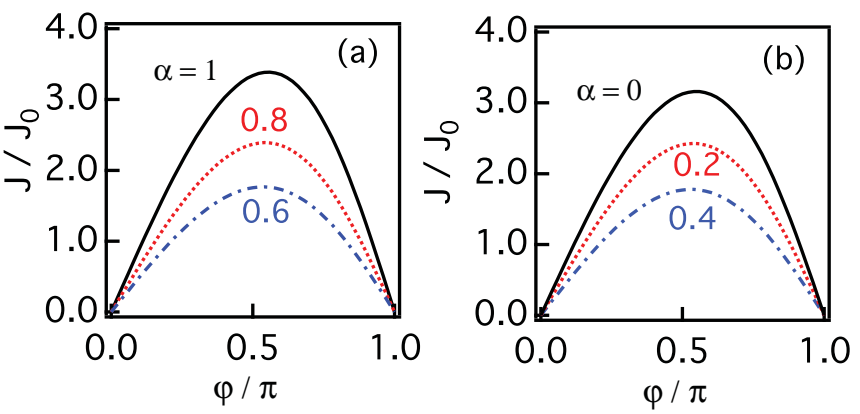

FIG. 5. (Color online) Current-phase relationships in Figs. 4(b) and $4(\mathrm{~d})$ calculated for a higher temperature $\left(T=0.1 T_{c}\right)$.

a higher temperature at $T=0.1 T_{c}$. In both Figs. 4(a) and 4(b), the CPR deviates from the sinusoidal relation only slightly at $T=0.1 T_{c}$. On the other hand, the amplitudes of the Josephson current remain at a sufficiently larger value than $J_{0}$.

\section{C. $d+p$ II}

Finally, we show the calculated results of the Josephson current for the $d+p$ II mixture as shown in Fig. 6. The temperature dependence of critical currents for several $\alpha$ satisfying $\Delta_{\mathrm{S}}>\Delta_{\mathrm{T}}$ indicates a strong low-temperature anomaly, as shown in Figs. 6(a) and 6(b). The critical current increase with decreasing temperature as $T^{-1}$ and the CPR at a low temperature shows the jump at $\varphi=\pi$. The zero-energy surface bound states are possible for all $\gamma$, as shown in Fig. 2(d). The presence of the flat zero-energy states explains the similarity of the results in Figs. 6(a) and 6(b) to those shown in Figs. 4(a) and 4(b), respectively. The calculated results for $\alpha=0.4,0.2$, and 0 satisfying $\Delta_{\mathrm{S}}<\Delta_{\mathrm{T}}$ are shown in Figs. 6(c) and 6(d). In
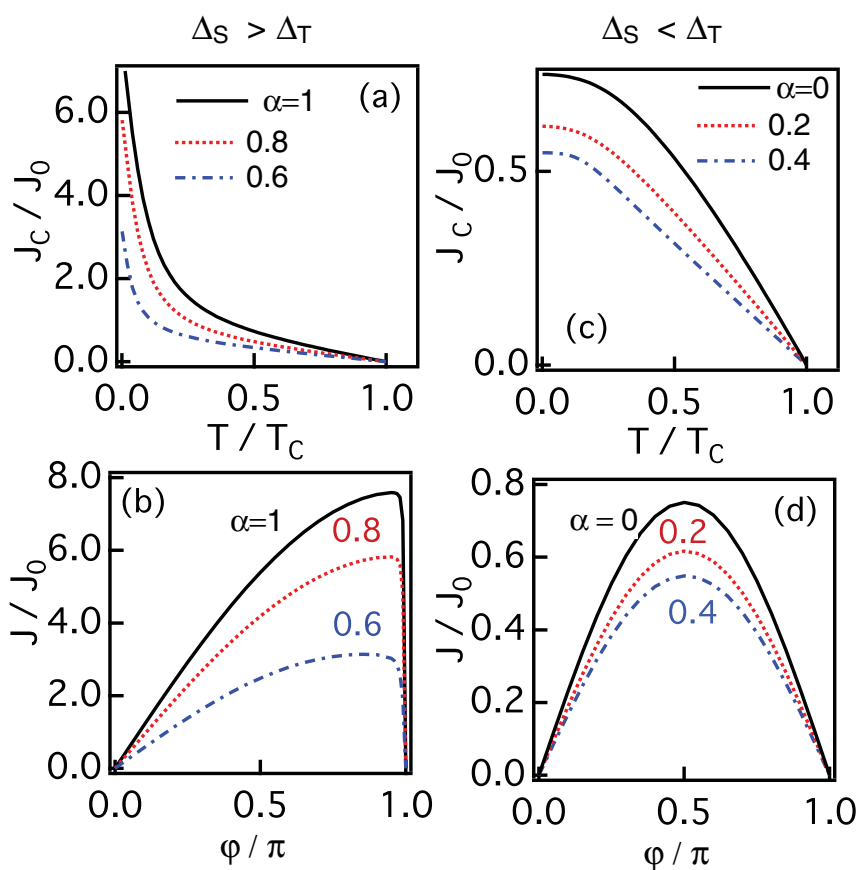

FIG. 6. (Color online) Results for $d+p$ II. The critical Josephson current is plotted as a function of temperature in (a) and (c). The current-phase relationship is shown at low temperature $(T=$ $\left.0.001 T_{c}\right)$. contrast, the results for $\Delta_{\mathrm{S}}<\Delta_{\mathrm{T}}$ in Figs. 6(c) and 6(d) have properties similar to those in Figs. 3(a) and 3(b). Namely, the Josephson current saturates at low temperature and the CPR is sinusoidal at low temperature. The zero-energy state at $\sin \gamma=$ \pm 1 exists, as shown in Fig. 2(e). Although this zero-energy state appears as a result of the resonant Andreev reflection, ${ }^{22}$ it does not significantly affect the Josephson current. The wave number $\sin \gamma= \pm 1$ means $k_{x}=\cos \gamma=0$. Thus a quasiparticle does not have momenta in the current direction in the zero-energy state. When we consider huge spin-orbit coupling, it has been pointed out that ${ }^{13,46,47}$ the flat zero-energy states appear for $\sin \gamma>(1-2 \lambda / \mu)$. In such cases, the flat zero-energy state may cause the low-temperature anomaly. This statement, however, is still unclear in realistic junctions with a thick insulating barrier because the contribution of a quasiparticle with $\sin \gamma \approx \pm 1$ to the Josephson current becomes exponentially small. Within the approximation of $\lambda / \mu \ll 1$, there is no effective zero-energy state that causes the low-temperature anomaly for $\Delta_{\mathrm{S}}<\Delta_{\mathrm{T}}$. Therefore, the Josephson current in Figs. 6(c) and 6(d) shows qualitatively the same behavior as those in Figs. 3(a) and 3(b), respectively. Thus the characteristic feature of the Josephson current for $d+p$ II also qualitatively changes around the point $\Delta_{\mathrm{S}}=\Delta_{\mathrm{T}}$.

At $\Delta_{\mathrm{S}}=0$, the subgap state with linear dispersion appears around $\sin \gamma=0$, as mathematically shown in Eq. (18). This zero-energy state, however, is not a result of the resonant Andreev reflection ${ }^{22}$ but is a result of a node in the pair potential. Thus $\Delta_{\mathrm{S}}=0$ cannot be a critical point. Actually, the results for $\alpha=0$ in Fig. 6(c) show the saturation of the Josephson critical current at low temperature.

\section{CONCLUSION}

In summary, we have theoretically studied the Josephson current between two noncentrosymmetric superconductors based on the Bogoliubov-de Gennes equation and a general current formula. We have assumed three types of order parameters that consist of spin-singlet $\Delta_{S}$ and spin-triplet components $\Delta_{\mathrm{T}}$ at the same time. The Josephson current for $\Delta_{\mathrm{S}}>\Delta_{\mathrm{T}}$ shows clearly the different characteristic behavior from those for $\Delta_{\mathrm{S}}<\Delta_{\mathrm{T}}$ for all pairing symmetries. The clear difference can be understood by analyzing the topologically protected zero-energy states at a surface of the noncentrosymmetric superconductor. The dispersionless zero-energy bound states are responsible for the strong low-temperature anomaly of the Josephson current in which the Josephson critical current increases as $1 / T$ with decreasing temperature. The surface state with linear dispersion causes the weak low-temperature anomaly in which the Josephson critical current increases logarithmically with decreasing temperature. When the surface zero-energy state is absent, the Josephson current obeys the Ambegaokar-Baratoff formula.

\section{ACKNOWLEDGMENTS}

This work was supported by KAKENHI (Grant No. 22540355) and the "Topological Quantum Phenomena" Grantin Aid (No. 22103002) for Scientific Research on Innovative Areas from the Ministry of Education, Culture, Sports, Science, and Technology (MEXT) of Japan. 
${ }^{1}$ E. Bauer, G. Hilscher, H. Michor, Ch. Paul, E. W. Scheidt, A. Gribanov, Yu. Seropegin, H. Noël, M. Sigrist, and P. Rogl, Phys. Rev. Lett. 92, 027003 (2004).

${ }^{2}$ K. Togano, P. Badica, Y. Nakamori, S. Orimo, H. Takeya, and K. Hirata, Phys. Rev. Lett. 93, 247004 (2004).

${ }^{3}$ M. Nishiyama, Y. Inada, and G.-Q. Zheng, Phys. Rev. B 71, 220505(R) (2005).

${ }^{4}$ P. A. Frigeri, D. F. Agterberg, A. Koga, and M. Sigrist, Phys. Rev. Lett. 92, 097001 (2004); P. A. Frigeri, D. F. Agterberg, and M. Sigrist, New J. Phys. 6, 115 (2004).

${ }^{5}$ M. Sato, Phys. Rev. B 73, 214502 (2006); M. Sato and S. Fujimoto, ibid. 79, 094504 (2009).

${ }^{6}$ Y. Tanaka, T. Yokoyama, A. V. Balatsky, and N. Nagaosa, Phys. Rev. B 79, 060505(R) (2009).

${ }^{7}$ A. B. Vorontsov, I. Vekhter, and M. Eschrig, Phys. Rev. Lett. 101, 127003 (2008).

${ }^{8}$ S. Fujimoto, J. Phys. Soc. Jpn. 76, 051008 (2007).

${ }^{9}$ Y. Yanase and M. Sigrist, J. Phys. Soc. Jpn. 77, 124711 (2008).

${ }^{10}$ J. Linder and A. Sudbo, Phys. Rev. B 76, 054511 (2007).

${ }^{11}$ C. Iniotakis, N. Hayashi, Y. Sawa, T. Yokoyama, U. May, Y. Tanaka, and M. Sigrist, Phys. Rev. B 76, 012501 (2007).

${ }^{12}$ C. K. Lu and S. Yip, Phys. Rev. B 80, 024504 (2009).

${ }^{13}$ Y. Tanaka, Y. Mizuno, T. Yokoyama, K. Yada, and M. Sato, Phys. Rev. Lett. 105, 097002 (2010).

${ }^{14}$ K. Yada, S. Onari, Y. Tanaka, and J. I. Inoue, Phys. Rev. B 80, 140509(R) (2009).

${ }^{15}$ N. Reyren et al., Science 317, 1196 (2007).

${ }^{16}$ P. M. R. Brydon, A. P. Schnyder, and C. Timm, Phys. Rev. B 84, 020501(R) (2011); A. P. Schnyder and S. Ryu, ibid. 84, 060504(R) (2011).

${ }^{17}$ G. Annunziata, M. Cuoco, C. Noce, A. Sudbo, and J. Linder, Phys. Rev. B 83, 060508(R) (2011).

${ }^{18}$ L. J. Buchholtz and G. Zwicknagl, Phys. Rev. B 23, 5788 (1981).

${ }^{19}$ J. Hara and K. Nagai, Prog. Theor. Phys. 74, 1237 (1986).

${ }^{20}$ C. R. Hu, Phys. Rev. Lett. 72, 1526 (1994).

${ }^{21}$ Y. Tanaka and S. Kashiwaya, Phys. Rev. Lett. 74, 3451 (1995); S. Kashiwaya and Y. Tanaka, Rep. Prog. Phys. 63, 1641 (2000).

${ }^{22}$ Y. Asano, Y. Tanaka, and S. Kashiwaya, Phys. Rev. B 69, 134501 (2004).

${ }^{23}$ S. Kashiwaya, Y. Tanaka, M. Koyanagi, H. Takashima, and K. Kajimura, Phys. Rev. B 51, 1350 (1995).

${ }^{24}$ J. Y. T. Wei, N.-C. Yeh, D. F. Garrigus, and M. Strasik, Phys. Rev. Lett. 81, 2542 (1998).
${ }^{25}$ A. Biswas, P. Fournier, M. M. Qazilbash, V. N. Smolyaninova, H. Balci, and R. L. Greene, Phys. Rev. Lett. 88, 207004 (2002).

${ }^{26}$ I. Iguchi, W. Wang, M. Yamazaki, Y. Tanaka, and S. Kashiwaya, Phys. Rev. B 62, R6131 (2000).

${ }^{27}$ B. Chesca, M. Seifried, T. Dahm, N. Schopohl, D. Koelle, R. Kleiner, and A. Tsukada, Phys. Rev. B 71, 104504 (2005); M. Wagenknecht, D. Koelle, R. Kleiner, S. Graser, N. Schopohl, B. Chesca, and A. Tsukada, S. T. B. Goennenwein, R. Gross, Phys. Rev. Lett. 100, 227001 (2008); N. P. Armitage, P. Fournier, and R. L. Greene, Rev. Mod. Phys. 82, 2421 (2010).

${ }^{28}$ C. L. Kane and E. J. Mele, Phys. Rev. Lett. 95, 146802 (2005).

${ }^{29}$ X.-L. Qi, T. L. Hughes, S. Raghu, and S.-C. Zhang, Phys. Rev. Lett. 102, 187001 (2009).

${ }^{30}$ Y. Tanaka and S. Kashiwaya, Phys. Rev. B 53, R11957 (1996).

${ }^{31}$ Y. S. Barash, H. Burkhardt, and D. Rainer, Phys. Rev. Lett. 77, 4070 (1996).

${ }^{32}$ Y. S. Barash, A. M. Bobkov, and M. Fogelström, Phys. Rev. B 64, 214503 (2001).

${ }^{33}$ Y. Asano and K. Katabuchi, J. Phys. Soc. Jpn. 71, 1974 (2002).

${ }^{34}$ H.-J. Kwon, K. Sengupta, and V. M. Yakovenko, Eur. Phys. J. B 37, 349 (2004).

${ }^{35}$ N. Hayashi, C. Iniotakis, M. Machida, and M. Sigrist, Physica C 468, 844 (2008).

${ }^{36}$ K. Borkje, Phys. Rev. B 76, 184513 (2007); K. Borkje and A. Sudbo, ibid. 74, 054506 (2006).

${ }^{37}$ R. P. Kaur, D. F. Agterberg, and M. Sigrist, Phys. Rev. Lett. 94, 137002 (2005)

${ }^{38}$ A. Sumiyama, K. Nakatsuji, Y. Tsuji, Y. Oda, T. Yasuda, R. Settai, and Y. Onuki, J. Phys. Soc. Jpn. 74, 3041 (2005).

${ }^{39}$ Y. Asano, Phys. Rev. B 64, 224515 (2001).

${ }^{40}$ V. Ambegaokar and A. Baratoff, Phys. Rev. Lett. 10, 486 (1963).

${ }^{41}$ F. Wilczek, Nature Phys. 5, 614 (2009).

${ }^{42}$ L. Fu and C. L. Kane, Phys. Rev. Lett. 100, 096407 (2008).

${ }^{43}$ L. Fu and C. L. Kane, Phys. Rev. B 79, 161408(R) (2009).

${ }^{44}$ Y. Asano, Y. Tanaka, and N. Nagaosa, Phys. Rev. Lett. 105, 056402 (2010).

${ }^{45}$ P. A. Ioselevich and M. V. Feigelman, Phys. Rev. Lett. 106, 077003 (2011).

${ }^{46}$ K. Yada, M. Sato, Y. Tanaka, and T. Yokoyama, Phys. Rev. B 83, 064505 (2011).

${ }^{47}$ M. Sato, Y. Tanaka, K. Yada, and T. Yokoyama, Phys. Rev. B 83, 224511 (2011) 